Ferizi, Uran; Schneider, T.; Panagiotai, E.; Nedjati-Gilani, G.; Zhang, H.; WheelerKingshot, C.A.M; Alexander, D.C.; Ranking diffusion-MRI models within in-vivo human brain data. IEEE 10th International Symposium on Biomedical Imaging:From Nano to Macro, San Francisco, CA, USA, April 7-11, 2013, 676-679. 10.1109/ISBI.2013.6556565.

\title{
NOTE
}

\section{RANKING DIFFUSION-MRI MODELS WITH IN-VIVO HUMAN BRAIN DATA}

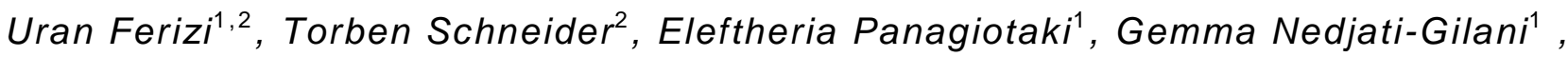
Hui Zhang ${ }^{1}$, Claudia A. M. Wheeler-Kingshott ${ }^{2}$, Daniel C. Alexander ${ }^{1}$

${ }^{1}$ Centre for Medical Image Computing and Department of Computer Science, University College London, United Kingdom

${ }^{2}$ NMR Research Unit, Queen Square MS Centre, Department of Neuroinflammation, UCL Institute of Neurology, London, United Kingdom

\section{ABSTRACT}

Diffusion MRI microstructure imaging provides a unique non-invasive probe into the microstructure of biological tissue. Its analysis relies on mathematical models relating microscopic tissue features to the MR signal. This work aims to determine which compartment models of diffusion MRI are best at describing the signal from in-vivo brain white matter. Recent work shows that three compartment models, including restricted intraaxonal, glial compartments and hindered extra-cellular diffusion, explain best multi b-value data sets from fixed rat brain tissue. Here, we perform a similar experiment using in-vivo human data. We compare one, two and three compartment models, ranking them with standard model selection criteria. Results show that, as with fixed tissue, three compartment models explain the data best, although simpler models emerge for the in-vivo data. We also find that splitting the scanning into shorter sessions has little effect on the models fitting and that the results are reproducible. The full ranking assists the choice of model and imaging protocol for future microstructure imaging applications in the brain.

Key words: Diffusion MRI, Brain imaging

\section{INTRODUCTION}

Diffusion MRI (dMRI) measures the water dispersion in biological tissue, which can be used to probe the microstructure. This technique is most often applied in the brain, especially where parallel fibres restrict the water mobility anisotropically, thus providing putative measures of white matter integrity and connectivity. Currently, the standard model for imaging diffusion in tissue is the diffusion tensor (DT) [1], which assumes a trivariate Gaussian dispersion pattern. Derived indices, e.g. mean diffusivity or fractional anisotropy, can correlate with major tissue 
damage, but lack the sensitivity and the specificity to provide to provide indices such as axon radius, density, orientation and permeability.

More complex models enable the estimation of more specific histological indices, such as axon radius, density, orientation, dispersion and permeability. Stanisz et al. [2] pioneered a multi-compartment representation of separate diffusive processes in nervous tissue. The Ball-Stick model [3] is the simplest possible two-compartment model with restricted axonal diffusion and isotropic extra-axonal diffusion. The Composite Hindered and Restricted Model of Diffusion (CHARMED) [4] is a similar model that allows anisotropic Gaussian Diffusion in the hindered extra-cellular space and cylindrical intra-cellular diffusion. The AxCaliber technique [5] extended CHARMED to estimate the distribution of axon diameters. Barazany et al.[6] demonstrated the approach in-vivo on a rat, adding a third compartment to account for cerebrospinal fluid (CSF) contamination. Alexander et al. [7] used a similar model to derive orientationally invariant indices of axon diameter and density. Recently, another model, NODDI [8], aims at capturing fiber density and dispersion.

The increasing model complexity can potentially lead to overfitting and false parameter estimation. Panagiotaki et al. [9] compared 47 diffusion MRI compartment models using data from fixed white matter (WM) of rats, via the Bayesian Information Criterion (BIC). After proposing a taxonomy of one, two and three compartment models, drawn from previous studies [2, 3, 4, 5, 6, 7, 8], they concluded that the three-compartment models perform best and DT worst. In this taxonomy, the first compartment, hindered in 3D, can be: a Tensor (full DT), a Zeppelin (cylindrically symmetric DT) or a Ball (isotropic DT). The second compartment, 'restricted' in 2D but free in the other direction (anisotropic restriction), can be: a Stick (oriented line) or a Cylinder (non-zero radius). The third compartment, isotropically restricted, can be: a Dot (bound fluid), a Sphere (where diffusion is restricted to within a nonzero radius), Astrosticks (Sticks isotropically distributed in 3D) or Astrocylinders (Cylinders in 3D).

Here, we perform a similar experiment in-vivo, on a human brain, using a rich, massively multi-shell High Angular Resolution Diffusion Imaging (HARDI) protocol. We com-

\section{$\rightarrow$ Page 677}

pare the models using BIC, and confirm with the Akaike Information Criterion (AIC). We find that, compared with the fixed tissue study [9], simpler three compartment models emerge. In addition, the ranking is consistent over datasets that are sampled through the Jackknife method.

\section{METHODS}

This section describes the acquisition protocol for our data and outlines the preprocessing we do to obtain a set of measurements for fitting. It then details the fitting procedure, the techniques we use for comparing the models and, lastly, the robustness of the ranking. 


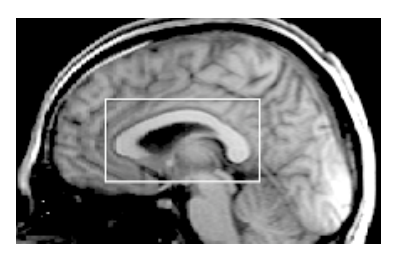

Fig. 1. Boxed is the scan volume

\subsection{Data acquisition and preprocessing}

Using a Pulsed Gradient Spin Echo sequence, on a 3T Phillips scanner, and having obtained ethical approval, we scan a 31 - yr old man in two separate non-stop sessions, each 4hrs long; we call this the $2 \times 4 \mathrm{hr}$ dataset. We then repeat this protocol in eight sessions lasting $1 \mathrm{hr}$; we call this the $8 \times 1 \mathrm{hr}$ dataset.

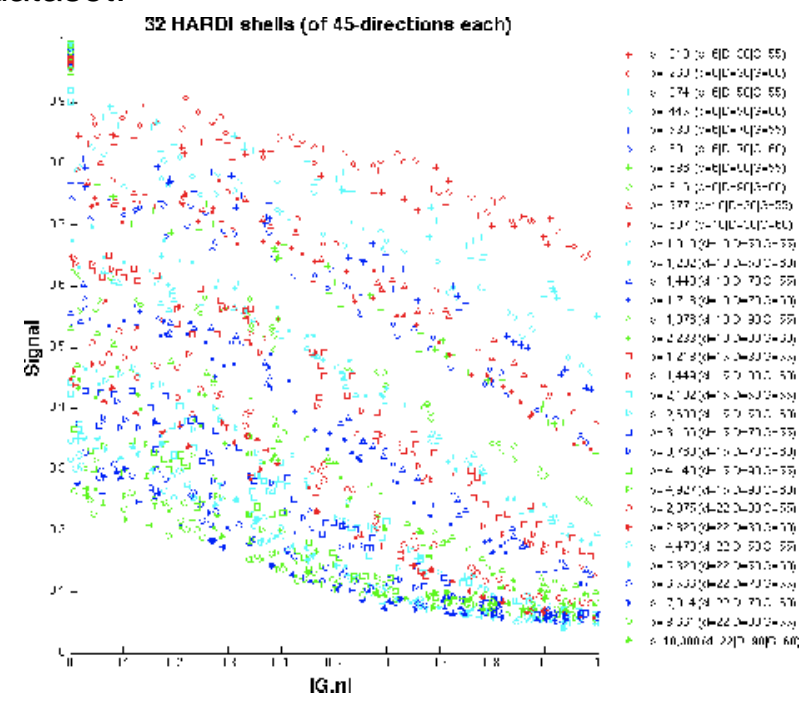

Fig. 2. The acquired signal. Legend: b-value (6 ||| G|)

The protocol uses 32 shells of 45 -directions each, which are randomly rotated to enhance the angular resolution. Each shell is identified by a combination of these dMRI parameters: gradient strength $|\mathrm{G}|=55,60 \mathrm{mT} / \mathrm{m}$, pulse width $6=6,10,15,22 \mathrm{~ms}$, pulse duration $=30,50,70,90 \mathrm{~ms}$, and has three interwoven $b=0$ acquisitions. The $b$-values thus range from 218 to $10,308 \mathrm{~s} / \mathrm{mm}^{2}$, with effective diffusion time in the range 28 to $82 \mathrm{~ms}$.

There are nine $4 \mathrm{~mm}$ thick sagittal slices, acquired with ZOOM-EPI with a reduced field-of-fiew (FOV) technique [10]. The FOV is centred on the mid-sagittal slice of the Corpus Callosum (CC), to which we assume the coherent CC fibres are perpendicular. The image size is $64 \times 64$ and the in-plane resolution $2 \mathrm{~mm} \times 2 \mathrm{~mm}$.

After manually segmenting the CC (shown in Fig.1), we select all voxels with FA>0.5 and principal eigenvector within $5^{\mathrm{O}}$ of the fibre direction. We create a single dataset by averaging the voxels satisfying these conditions. Fig.2 shows the rich coverage of the sampling space. We 
ignore any signal below the observed noise floor of 0.1 . Before fitting the models, the signal of each DW slice is normalised by the corresponding diffusion-unweighted $(b=0)$ measurement with the same echo time, to remove T2 effects.

\subsubsection{Model Fitting}

We fit the 32 models of diffusion (listed in Table 1) to the signal, via the open source software tool Camino [11]. Each model is fitted 1000 times to the data, using the LevenbergMarquardt algorithm with a perturbed starting point, to extract the parameters that produce the minimum objective function. This ensures we avoid local minima and obtain the best-fit parameters. The fitting uses an offset-Gaussian noise model, as in [9], to construct the objective function:

$$
\left.\frac{\left(\mathrm{Si}^{\mathrm{i}} \mathbf{}-\mathrm{S} 2\right.}{2 \sigma 2} \underline{\mathbf{i}}+\sigma^{2}\right)^{2}
$$

where $\mathrm{N}=32^{*}(45+3)$ is the total number of measurements, "Si is the $\mathrm{i}$-th measured signal, $\mathrm{Si}$ its prediction from the model; $\sigma 2$ is the signal variance, which we estimate a priori. This objective function accounts for bias introduced by the Rician noise inherent in the MRI data in a simplistic way $[12,13]$ that is more numerically stable than a full Rician log-likelihood objective function.

\subsubsection{Model Selection}

We use these model selection criteria:

$$
\begin{gathered}
\mathrm{BIC}=-2 \log (\mathrm{L})+\mathrm{K} \log (\mathrm{N}) \\
\mathrm{AIC}=-2 \log (\mathrm{L})+2 \mathrm{~K}
\end{gathered}
$$

where $L$ is the likelihood of obtaining the present data given the model and $K$ is the number of free parameters. These criteria quantify the trade-off goodnessof-fit, so as to identify the simplest model The lower the value, the more predictive the is less conservative in penalising complexity.

$$
\begin{gathered}
\text { between complexity and } \\
X_{i=1}^{N} \text { that explains the data. } \\
\text { model is. In general, AIC }
\end{gathered}
$$

\section{Page $678 \rightarrow$}

\section{RESULTS}

Table 1 shows the models' BIC/AIC/LSE ranking for both datasets. The three compartment models come out top for both the $2 \times 4 \mathrm{hr}$ and $8 \times 1 \mathrm{hr}$ dataset, as in [9]. Within this category of models, the Zeppelin/Tensor hindered compartments outperform Ball, and the ranking shows a preference for Dot/Sphere over Astrosticks and Astrocylinders; because of its simplicity, the Stick is slightly preferred by BIC and AIC over the Cylinder. As in [9], the DT comes out as the worst model.

Fig. 3 shows the consistency of these BIC rankings. Here, we construct 100 Jackknife dataset samples from each dataset, each time picking half the number of measurements at random from the original dataset. The rankings are broadly similar, although the $8 \times 1 \mathrm{hr}$ stability matrix shows less consistency in the ranking.

Fig.4 illustrates why three compartment models explain the data better than one or two 
compartment models. Similarly, we see that within three-compartment models, an anisotropic hindered compartment improves the fitting.

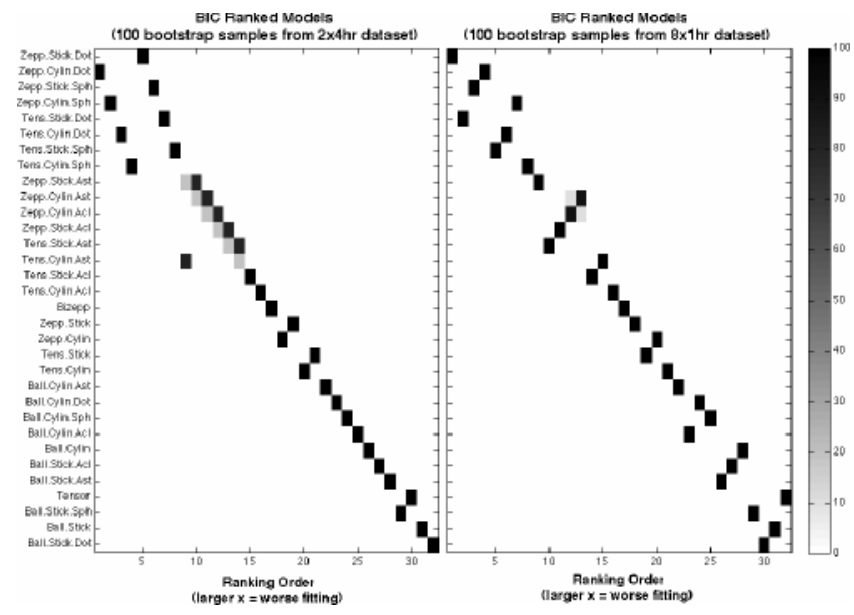

Fig. 3. Each model's ranking stability for 100 Jackknife samples from the $2 \times 4 \mathrm{hr}$ data (left matrix) and $8 \times 1 \mathrm{hr}$ data (right matrix). In the matrices, the frequency of every ranking ( $\mathrm{x}$-axis) is given by $2 \times 4 h r$ dataset $8 \times 1 \mathrm{hr}$ dataset

\begin{tabular}{c|cccc|ccc}
$\begin{array}{c}\text { Compartment } \\
\text { Models }\end{array}$ & $\begin{array}{c}\text { BIC } \\
\text { score }\end{array}$ & $\begin{array}{c}\text { BIC } \\
\text { rank }\end{array}$ & $\begin{array}{c}\text { AIC } \\
\text { rank }\end{array}$ & $\begin{array}{c}\text { LSE } \\
\text { rank }\end{array}$ & $\begin{array}{c}\text { BIC } \\
\text { rank }\end{array}$ & $\begin{array}{c}\text { AIC } \\
\text { rank }\end{array}$ & $\begin{array}{c}\text { LSE } \\
\text { rank }\end{array}$ \\
Zeppelin-Stick-Dot & 659.4 & 1 & 1 & 3 & 1 & 4 & 5 \\
Zeppelin-Cylinder-Dot & 666.6 & 2 & 2 & 4 & 2 & 5 & 6 \\
Zeppelin-Stick-Sphere & 666.8 & 3 & 3 & 5 & 3 & 6 & 7 \\
Tensor-Stick-Dot & 672.3 & 4 & 4 & 1 & 4 & 1 & 1 \\
Zeppelin-Cylinder-Sphere & 678.2 & 5 & 7 & 7 & 7 & 8 & 8 \\
Tensor-Cylinder-Dot & 679.5 & 6 & 5 & 2 & 5 & 2 & 2 \\
Tensor-Stick-Sphere & 681.6 & 7 & 6 & 6 & 6 & 3 & 3 \\
Tensor-Cylinder-Sphere & 695.3 & 8 & 8 & 8 & 8 & 7 & 4 \\
Zeppelin-Sick-Astrosticks & 789.3 & 9 & 9 & 13 & 9 & 11 & 13 \\
Zeppelin-Stick-Astrocylinders & 796.7 & 10 & 10 & 14 & 11 & 15 & 15 \\
Zeppelin-Cylinder-Astrosticks & 796.7 & 11 & 11 & 15 & 10 & 14 & 14 \\
Zeppelin-Cylinder-Astrocylinders & 797.1 & 12 & 13 & 16 & 12 & 16 & 16 \\
Tensor-Stick-Astrosticks & 802.1 & 13 & 12 & 9 & 13 & 9 & 9 \\
Tensor-Stick-Astrocylinders & 809.4 & 14 & 14 & 11 & 15 & 12 & 12 \\
Tensar-Cylinder-Astrosticks & 809.5 & 15 & 15 & 12 & 14 & 10 & 10 \\
Tensor-Cylinder-Astrocylinders & 816.6 & 16 & 16 & 10 & 16 & 13 & 11 \\
Bizepp & 885.9 & 17 & 17 & 17 & 17 & 17 & 17 \\
Zeppelin-Stick & 910.0 & 18 & 18 & 20 & 18 & 21 & 22 \\
Zeppelin-Cylin & 917.2 & 19 & 19 & 21 & 21 & 22 & 21 \\
Tensor-Stick & 923.0 & 20 & 20 & 18 & 20 & 18 & 19 \\
Tensor-Cylin & 930.3 & 21 & 21 & 19 & 22 & 19 & 18 \\
Ball-Cylinder-Astrosticks & 1176.5 & 22 & 22 & 22 & 19 & 20 & 20 \\
Ball-Cylinder-Astrocylinders & 1260.2 & 23 & 23 & 23 & 25 & 25 & 25 \\
Ball-Cylinder-Dot & 1266.4 & 24 & 24 & 24 & 23 & 23 & 23 \\
Ball-Cylinder-Sphere & 1277.7 & 25 & 25 & 25 & 24 & 24 & 24 \\
Ball-Cylin & 1298.5 & 26 & 26 & 26 & 26 & 27 & 27 \\
Ball-Stick-Astrocylinders & 1422.5 & 27 & 27 & 27 & 27 & 26 & 26 \\
Ball-Stick-Astrosticks & 1427.7 & 28 & 28 & 28 & 28 & 28 & 28 \\
Ball-Stick-Sphere & 1461.0 & 29 & 29 & 29 & 29 & 29 & 29 \\
Ball-Stick & 1469.1 & 30 & 30 & 30 & 30 & 31 & 31 \\
Baal-Stick-Dot & 1476.4 & 31 & 31 & 31 & 31 & 30 & 30 \\
Tensor & 1907.9 & 32 & 32 & 32 & 32 & 32 & 32
\end{tabular}

Thbofe 1. The ranking of the models using both datasets. In
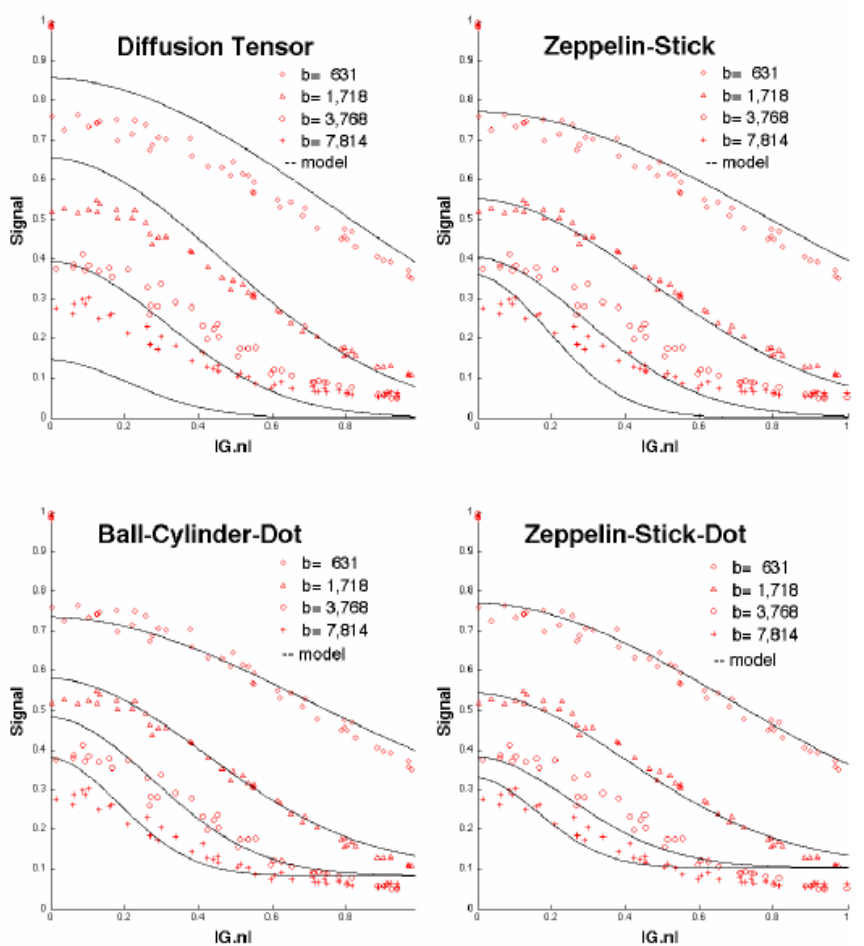
addition, the actual computed score for BIC is given for the $2 \times 4$ hr dataset, by which the models are ordered. LSE is the objektive Function. 
To explore more specific microstructure parameters, we sampled a wide range of achievable bvalues and diffusion times on a clinical system. The ranking we obtain is similar to previous observations from fixed tissue [9], with minor differences. The fixed-tissue study's 9.4T pre-clinical scanner used much stronger gradients, i.e. much shorter pulses, which makes the acquisition much more sensitive to the size of smaller axons. In this study, however, our protocol employs a higher angular resolution, which may significantly improve more complex models.

We emphasize that the choice of models our analysis suggests is not appropriate for existing sparse data sets such as off-the-shelf single shell HARDI data, which only support simple models. Rather, these results inform the choices for future in-vivo microstructure imaging once we identify the right model. Experiment design techniques such as [14] can determine economical protocols. Conversely, the protocol we use here is designed specifically for model selection rather than large-scale application. Another limitation is that this model selection is performed with data from only a part of the CC, which is relatively homogeneous, with little fibre crossing or CSF contamination, so other models may perform better away from the CC (e.g. with a CSF pool as in $[6,8])$.

Future work will seek to reproduce these results across other subjects, and find an economical protocol that identifies the same top model as the full one. We also plan to include more models with, e.g., a distribution of pore sizes [5, 9] or orientation dispersion [8].

\section{ACKNOWLEDGEMENTS}

Thanks to EPSRC for funding the research studentship of U.Ferizi.

We are grateful to the Multiple Sclerosis Society of Great Britain and Northern Ireland for supporting the NMR unit.

This work was undertaken at UCLH/UCL who are part- funded by the Department of Health NIHR Biomedical Research Centres funding scheme.

\section{REFERENCES}

[1] P.J. Basser, J. Mattiello, and D. LeBihan, "MR diffusion tensor spectroscopy and imaging," Biophysical journal, vol. 66, no. 1, pp. 259-267, 1994.

[2] G.J. Stanisz, G.A. Wright, R.M. Henkelman, and A. Szafer, "An analytical model of restricted diffusion in bovine optic nerve," MRM, 1997.

[3] T.E.J. Behrens, M.W. Woolrich, M. Jenkinson, H. Johansen-Berg, R.G. Nunes, S. Clare, P.M. Matthews, J.M. Brady, and S.M. Smith, "Characterization and propagation of uncertainty in diffusion- weighted MR imaging," MRM, 2003.

[4] Y. Assaf, R.Z. Freidlin, G.K. Rohde, and P.JFig. 4. Synthesised signal from three representative models Basser, "New modeling and experimental framework to characterize hindered and (solid line) with raw data (marked red) for 4 shells only. $G$ is the gradient unit vector; $\mathrm{n}$ is the fibre unit vector. restricted water diffusion in brain white matter," MRM, vol. 52, no. 5, pp. 965-978, 2004.

[5] Y. Assaf, T. Blumenfeld-Katzir, Y. Yovel, and P.J. Basser, "Axcaliber: a method for 
measuring axon diameter distribution from diffusion MRI," $M R M, 2008$.

[6] D. Barazany, P.J. Basser, and Y. Assaf, "In vivo measurement of axon diameter distribution in the corpus callosum of rat brain," Brain, p. 1210, 2009.

[7] D.C. Alexander, P.L. Hubbard, M.G. Hall, E.A. Moore, M. Ptito, G.J.M. Parker, and T.B. Dyrby, "Orientation- ally invariant indices of axon diameter and density from diffusion MRI," Neurolmage, 2010.

[8] H. Zhang, P.L. Hubbard, G.J.M. Parker, and D.C. Alexander, "Axon diameter mapping in the presence of orientation dispersion with diffusion MRI," Neurolmage, 2011.

[9] E. Panagiotaki, T. Schneider, B. Siow, M.G. Hall, M.F. Lythgoe, and D.C. Alexander, "Compartment models of the diffusion MR signal in brain white matter: A taxonomy and comparison," Neurolmage, 2011.

[10] B.J. Wilm, "Reduced field-of-view MRI using outer volume suppression for spinal cord diffusion imaging," MRM, 2007.

[11] P.A. Cook, Y. Bai, S. Nedjati-Gilani, K.K. Seunarine, M.G. Hall, G.J. Parker, and D.C. Alexander, "Camino: Open-source diffusion MRI reconstruction and processing," in 14th scientific meeting of the ISMRM, 2006.

[12] Daniel C. Alexander, ," in Visualization and Processing of Tensor Fields, Joachim Weickert and Hans Hagen, Eds., pp. 83-106. Springer, 2006.

[13] D.K. Jones and P.J. Basser, "'Squashing peanuts and smashing pumpkins': How noise distorts DW-MR data," MRM, 2004.

[14] D.C. Alexander, "A general framework for experiment design in diffusion MRI and its application in measuring direct tissue-microstructure features," MRM, 2008. 